\title{
Introduction to supersymmetric spin networks
}

\author{
Yi Ling * \\ Center for Gravitational Physics and Geometry \\ Department of Physics \\ The Pennsylvania State University \\ University Park, PA, USA 16802 \\ and \\ The Blackett Laboratory \\ Imperial College of Science, Technology and Medicine \\ South Kensington, London SW7 2BZ, UK
}

October 23, 2018

\begin{abstract}
In this paper we give a general introduction to supersymmetric spin networks. Its construction has a direct interpretation in context of the representation theory of the superalgebra. In particular we analyze a special kind of spin networks with superalgebra $O s p(1 \mid 2 n)$. It turns out that the set of corresponding spin network states forms an orthogonal basis of the Hilbert space $\mathcal{L}^{2}(\mathcal{A} / \mathcal{G})$, and this argument holds even in the q-deformed case. The $O s p(n \mid 2)$ spin networks are also discussed briefly. We expect they could provide useful techniques to quantum supergravity and gauge field theories from the point of non-perturbative view.
\end{abstract}

\section{Introduction}

The notion of spin networks originally was advocated by Roger Penrose in 1970s when he tried to give a quantum mechanical description of the geometry of space[1]. In his opinion,

*email: *ling@phys.psu.edu 
the final version of quantum geometry should be a combinatorial theory in which we consider the different combinations and permutations of objects such that we could derive the discrete spectra of observables in the quantum mechanical level. After that the idea of spin networks was introduced to many areas, including lattice gauge theory[2] and topological field theory[3]. In the middle of 1990s, the spin networks was introduced in loop quantum gravity in a quite different way 4, 5]. It was exploited to construct the Hilbert space of kinematical quantum states and consequently the discrete spectra of the area and volume of the space were obtained [6]. Later the dynamics of the spin networks were also considered and its evolution gives rise to casual set of spin networks [7] or spin foams [8].

It's evident to see the importance of spin networks if we list some basic features it contains. First spin networks is a very general notion in quantum field theory in which gauge fields involve. In particular they are gauge invariant objects, in the sense that the corresponding spin network states will be solutions to the Gauss constraint naturally if we take the standard Dirac procedures to quantize the theory. As a result, it would be much easier to find the physically related subspace in Hilbert space. In path integral formulation, we can consider the functional integration on the modular space since a well-defined measure theory can be established in context of spin networks. In loop quantum gravity, they are background independent and non-perturbative objects as well.

Until now we mainly focus on $S U(2)$ spin networks since it has important application to quantum general relativity [6, 8] ]. However, in principle we could construct the spin networks associated to other groups or supergroups. More importantly we have a belief that they could be applied to quantum supergravity and gauge theories as well. Based on this motivation, the supersymmetric spin networks firstly was introduced in 10 and then was developed in [11]. In [10] by virtue of supersymmetric spin networks, we carry out a nonperturbative quantization of simple supergravity, in particular we find the spectrum of area operator taking a discrete form,

$$
\hat{A}\left|\Gamma^{s g}, e_{i}, v_{j}\right\rangle=\sum_{i} l_{p}^{2} \sqrt{j_{i}\left(j_{i}+\frac{1}{2}\right)}\left|\Gamma^{s g}, e_{i}, v_{j}\right\rangle,
$$

where $l_{p}$ is the Planck length and $j_{i}=\frac{n_{i}}{2}$.

\footnotetext{
${ }^{1}$ We also notice that spin networks with other Lie groups have also been discussed in some places 9 .
} 
In this paper we develop the construction of supersymmetric spin networks. After giving an overview on some general features of spin networks in section two, we study a special kind of super spin networks which has superalgebras $O s p(1 \mid 2 n)$ in the consequent section, and $O \operatorname{sp}(N \mid 2)$ spin networks is also discussed briefly in section four. In section five we discuss some possible applications of super spin networks.

\section{An overview}

Spin network is a graph, $\Gamma\left(e_{i}, v_{j}\right)$, embedded in a three dimensional manifold $M$, which is composed of edges (or links) and vertices (either nodes or joints). To each edge, we assign the color, $e_{i}$, which is related to the labels of the irreducible representation of groups, and to each vertex, we assign an intertwiner operator, $v_{j}$, which maps the incoming irreducible representations to the outcoming ones at the vertex.?

The construction of spin networks has a direct interpretation in representation theory of groups. In spin networks, each edge $e$ labeled by the representation could be understood as a parallel propagator or holonomy of the connection $A, U_{e}(A)$, along the edge in connection representation. In matrix notation of group theory, it also corresponds to the higher dimensional irreducible representation of the group element. In $S U(2)$ spin networks, we also think of the edge as the combination of many ropes, each of which corresponds to the fundamental presentation of the group. Therefore the reason that each edge can be decomposed into many ropes stems from the fact that every higher dimensional irreducible representation of the group can be obtained by employing the symmetrization or anti-symmetrization procedures from the fundamental representations. It is this fact that we can decompose the spin network into multi-loop graphs by permuting and connecting all the ropes to form loops, and finally we are able to establish the transformation between spin network states and loop states in the corresponding Hilbert space.

Intertwiner operators associated with every vertex in spin network can be understood as the different ways that we could carry out to connect the ropes when edges meet at the same vertex. Correspondingly, in the language of representation theory of groups, it

\footnotetext{
${ }^{2}$ we adopt the convention that the vertex will be called k-valent one if there are $k$ edges meeting at this vertex.
} 
corresponds to the fact that tensor products of several irreducible representations can be completely decomposed into the direct sum of the irreducible representations. In hence they are invariant tensors in irreducible representations of groups and given by standard ClebschGordan $(C G)$ theory. In the case of $S U(2)$ spin networks, when the vertex is a tri-valent one, the decomposition of the tensor product is unique. If the edges are more than three, we then can divide the multi-valent vertex into the tri-valent vertices by making use of the intertwiner operator. At the same time, restricted by the expansion of Clebsch-Gordan series, the colors associated with edges which meet at the same vertex must satisfy some conditions consistent with these $C G$ series. We call them admissible conditions. For instance, consider three valences with colors $(a, b, c)$ meet at the same vertex in ordinary $S U(2)$ spin networks, then they have to satisfy the triangle inequality and the sum of them has to be even numbers, however, in the case of $O s p(1 \mid 2)$ then the sum can be any positive integer.

Associated with each spin network, we can obtain one number by taking the trace of corresponding matrix product of the propagators along edges in representation space, which is called the evaluation of spin networks. It has very important applications to quantum gravity. In particular when we consider the action of operators such as area and volume observables on the spin network states, it provides us a practical way to work out the spectra of these observables. In cases of $S U(2)$ and $O s p(1 \mid 2)$, the evaluations of spin networks, in particular the theta graphs, are discussed respectively in [12, 10], where $6 j$ symbols and recoupling theories play important roles.

Based on the spin network $\Gamma\left(e_{i}, v_{j}\right)$, we can define a spin network state, $\Phi_{f_{\Gamma}}(A)$, by means of the cylindrical function with the form

$$
\Phi_{f_{\Gamma}}(A):=f_{\Gamma}\left(U_{e_{1}}(A), \ldots, U_{e_{n}}(A)\right)
$$

where cylindrical function $f_{\Gamma}$ refers to taking the holonomy along each edge and then contracting the holonomy matrix with the intertwiners at each vertex where edges meet. Spin network states have more advantages than loop states since they are linear independent and do form a basis of the Hilbert space, rather than loop states the space of which is over completed and contains some identities. To show the spin network states form a basis in the Hilbert space, We need solve two key problems. One is the definition of inner product of the spin network state, consequently we can show any two different spin network states are 
orthogonal and linear independent; the other one is the completeness of the spin network states, namely any state in Hilbert space can be expressed in terms of spin network states. In the case of $S U(2)$, these two problems are solved successfully mainly by virtue of the Haar measure and Peter-Weyl theorem in group theory respectively [4, 6, 6]. As a result, $S U(2)$ spin networks plays a key role to form a linear independent basis of the Hilbert space in loop quantum gravity.

When we try to extend the notion of spin networks to the supersymmetric case, we need construct the graphs and find the rules which must be completely consistent with the representation theory of superalgebras. Following constructions of $S U(2)$ and $O s p(1 \mid 2)$ spin networks [4, 10] which has been recalled above, let us list some basic procedures that we have to take into account in this paper.

- The definition of supersymmetric spin networks. At the first sight, it's simple to define the supersymmetric spin networks. we only need to change the representations associated to edges to the corresponding representations of the supergroups and label the vertex by intertwiner operator appropriately. But after that, to make the spin network well defined, we need to consider the following related questions.

- What's the admissible conditions associated with the trivalent vertex? Or equivalently, can any tensor product of the irreducible representations be decomposed into the direct sum of the irreducible representations? Due to the features of superalgebras their own, we will face some troubles at once, because unlike the Lie algebra, the tensor products of many kinds superalgebras are not completely reducible into the irreducible ones. We could see this trouble elsewhere when we construct the $O \operatorname{sp}(2 \mid 2)$ spin networks. [11]

- Could we find a way to evaluate the graphs such that we could consider the action of the operators on the corresponding spin network states and then calculate the spectra of the operators? More explicitly, can every edge be decomposed into ropes, as we do in the case of $S U(2)$ ? Namely, can any irreducible representation of the supergroups be constructed from the fundamental representation? We will also see only some special sorts of superalgebras have such features. Furthermore, is it possible to carry out a graphic representation of computing the enclosure of edges? 
- Does the set of the corresponding spin network states form a basis for the Hilbert space? To show this, first we need show that any different spin network states are orthogonal and linear independent, second any states in the space can be decomposed into the sum of the spin network states.

To answer these questions, in this paper we extend the strategy in $S U(2)$ spin networks to a special kind of supersymmetric spin network which is equipped with superalgebra $O s p(1 \mid 2 n)$. The analysis of this special one will lead to some general comments on the construction of supersymmetric spin networks with other superalgbras.

But before we do that, let us recall some basic facts related to superalgebras in the last part of this section. Superalgebras and supergroups were proposed in physics to construct the supersymmetric model in which bosons and fermions are placed in the same supermultiplet and they could change into each other under the supersymmetric transformation. In fact, every superalgebra or graded algebra contains two kinds of generators. One is even which is the bosonic part and the other is odd corresponding to fermionic part. Contrasting to the ordinary algebras, the even generators of the superalgebra are associated with the commuting parameters while the odd ones are associated with the anti-commuting parameters. A special kind of superalgebra is classical simple Lie superalgebra, whose odd part is completely reducible into one or two irreducible subspace. Its classification and representation is given in the fundamental paper by Kac 13. Furthermore, if classical superalgebras admit a nondegenerate metric tensor, it's called basic superalgebras, which is the closest one to simple Lie algebra. All irreducible representations of basic superalgebras are obtainable from a highest weight and the Schur lemma holds under the usual way. However, unlike the ordinary Lie algebras, normally superalgebras have two kinds of representations. One is typical, and the other one is atypical. The typical representations are irreducible and more like the ordinary representations of the Lie algebra, however the atypical ones are in many respects degenerate. In particular the atypical representations maybe are not completely reducible, for example if they occur as the semidirect sums of several irreducible atypical representations.

Many kinds of Lie superalgebras do not have properties similar to those of Lie algebras. For example, the complete reducibility is not valid to all the simple Lie superalgebra any 
more, even to basic ones. Finite reducible but indecomposable representations may appear if we consider the tensor products of irreducible representations. However, the representations of $O s p(1 \mid 2 n)$ have all the nice properties of those of semisimple Lie algebras. For example, its all reducible representations are fully reducible and therefore a generalized Wigner-Eckhart theorem holds.

The Lie supergroups are obtained by exponentiating Lie superalgebras. Particularly for supergroups $S U(N \mid M)$ and $O \operatorname{sp}(N \mid 2 M)$, it's known that all the representations constructible can be obtained from the direct product of fundamental representations.

\section{$3 \quad O \operatorname{sp}(1 \mid 2 n)$ spin networks}

\subsection{Definition}

First let us concentrate on a special kind of spin networks with superalgebra $O s p(1 \mid 2 n)=$ $B(0, n)$ [14], which is a subset of the orthosympletic Lie superalgebras $O s p(M \mid 2 n)$ ]. Its even part is $O(1) \otimes S p(2 n)$ and the Dynkin diagram is shown in Fig.(1). The finite dimensional

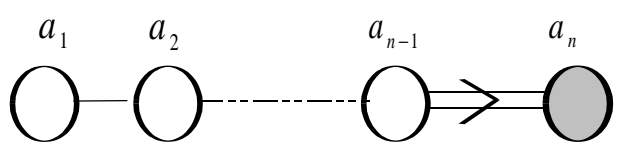

Figure 1: Dynkin diagram of $O s p(1 \mid 2 n)$

irreducible representation is characterized by its highest weight $\Lambda=\left(a_{1}, a_{2}, \ldots a_{n}\right)$ which takes the form

$$
\Lambda=a_{1} \omega_{1}+a_{2} \omega_{2}+\ldots a_{n} \omega_{n}
$$

where $\omega_{i}(i=1 \ldots n)$ is the fundamental weight and the coordinate $a_{i}(i=1 \ldots n)$ has to be nonnegative integer. The $S p(2 n)$ representation containing in the representation can be read out from the diagram directly by replacing the odd root $a_{n}$ by $b_{n}=\frac{1}{2} a_{n}$. Correspondingly, we assign the edge in $O s p(1 \mid 2 n)$ spin networks is labeled by $e_{i}$ which is defined as a partition

\footnotetext{
${ }^{3}$ The classification of superalgebra was established in [13]. There are three kinds of orthosympletic algebras $O s p(M \mid 2 n): B(m, n)=O s p(2 m+1 \mid 2 n) ; C(n)=O s p(2 \mid 2 n-2),(n \geq 2)$ and $D(m, n)=$ $O s p(2 m \mid 2 n),(m, n \geq 2)$.
} 
of $\left(a_{1}, \ldots, a_{n}\right)$. In the language of spin networks we also call the weight coordinates colors of the edge. In the simplest case of $n=1$, we see the representation of superalgebra is labeled by only one integer $e_{1}=2 j_{1}$, and the corresponding spin networks is discussed in detail in [10].

\subsection{Elements: edges and vertices}

As we mentioned above, in general there are two kinds of representations of the superalgebra. One is typical, and the other one is atypical. However, superalgebra $O s p(1 \mid 2 n)$ has a remarkable feature. It has only typical representation. The fundamental representation is labeled by weight coordinates $(1,0, \ldots, 0)$, and the basis vector of the representation is $2 n+1$ dimensional and contains one boson and $2 n$ fermions.

$$
\xi_{\alpha}=\left(\begin{array}{c}
\psi_{A} \\
\phi_{o}
\end{array}\right),
$$

where $\psi_{A}$ is the fermionic part of the representation and $A=(1, \ldots, 2 n)$ is the spinor index of $S p(2 n)$, while $\phi_{o}$ is the bosonic part which is only one dimensional. Then the unit element in the fundamental representation can be illustrated as fig.(2), in which we denote the $S p(2 n)$ element by the thin line and the single bosonic part by the dotted line.

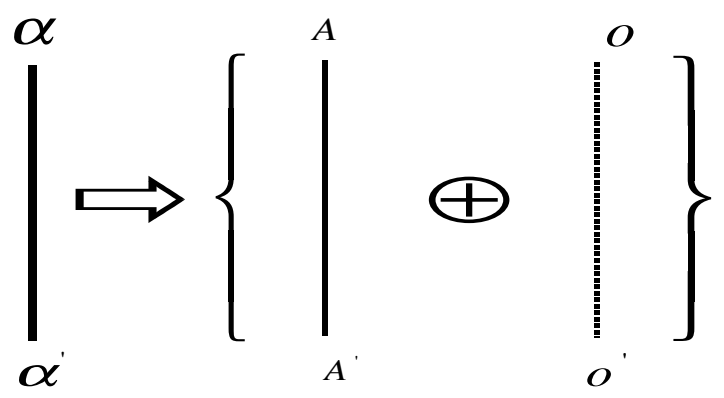

Figure 2: Composition of unit element with color $(1,0 \ldots, 0)$.

The higher finite dimensional representations of the superalgebra can be obtained by symmetrizing and anti-symmetrizing the fundamental representations 16]. Note that in the simplest case of $n=1$, we only need take the symmetrization procedures since the antisymmetrization of two spinor indices will be identical to the trivial representation. In context of Young tableaus, we have only one row of boxes. However, for $n \geq 2$, we need take 
both symmetrization and anti-symmetrization procedures to obtain all the finite dimensional irreducible representations. For instance, we consider the tensor products of two fundamental representations, we can symmetrize two basis vectors which is defined as, ff

$$
\xi_{(\alpha \beta)}=\xi_{\alpha}^{1} \xi_{\beta}^{2}+(-)^{g(\alpha) \cdot g(\beta)} \xi_{\beta}^{1} \xi_{\alpha}^{2}
$$

Also we can antisymmetrize them as

$$
\xi_{(\alpha \beta)}=\xi_{\alpha}^{1} \xi_{\beta}^{2}-(-)^{g(\alpha) \cdot g(\beta)} \xi_{\beta}^{1} \xi_{\alpha}^{2}
$$

where $g(\alpha)$ is the grade of the index. For fermionic indices, it's one and for bosonic it's zero. If we denote the symmetrization by square box in the graph, and antisymmetrization by a circle labeled by the number of ropes under consideration, then the unit elements of the supergroup under these representations are illustrated in Fig.(3).

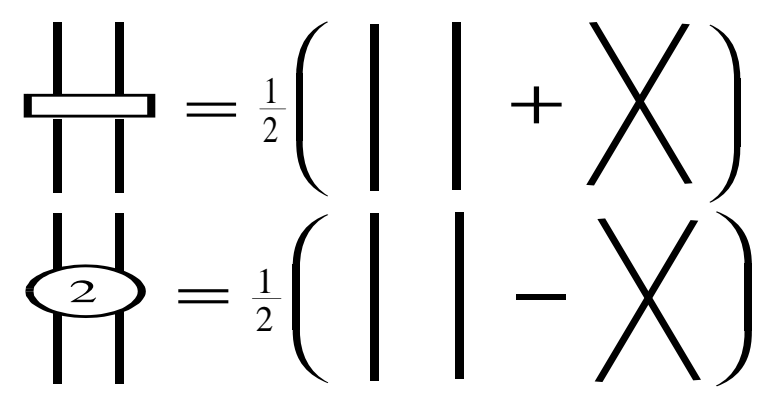

Figure 3: Symmetrization and anti-symmetrization of fundamental representation

Their weight coordinates are $(2,0, \ldots 0)$ and $(0,1,0, \ldots, 0)$ respectively. We also call the unit element symmetrizer in which ropes are symmetrized and antisymmetrizer in which ropes are antisymmetrized. Unlike the symmetrization, We can derive the following properties of the antisymmetrization in graphic representations, see fig.(伍), fig. (5) and fig. (6).

These properties are easily proved by using the definition of symmetrizers and antisymmetrizers shown in (3). For instance in fig.(5), when two lines are both symmetrized and

\footnotetext{
${ }^{4}$ Strictly speaking, unlike the superalgebra $S U(N \mid M)$, the symmetrized basis $\xi_{(\alpha \beta)}$ need to shift a little bit by the condition $G_{\alpha \beta} \xi_{(\alpha \beta)}=0$ [16], where $G_{\alpha \beta}$ is the invariant bilinear form taking the form $\left(\begin{array}{ccc}1 & 0 & 0 \\ 0 & 0 & I_{n} \\ 0 & -I_{n} & 0\end{array}\right)$. However this shift will not affect our construction of the unit element of the supergroups. We just ignore this shift in this paper.
} 


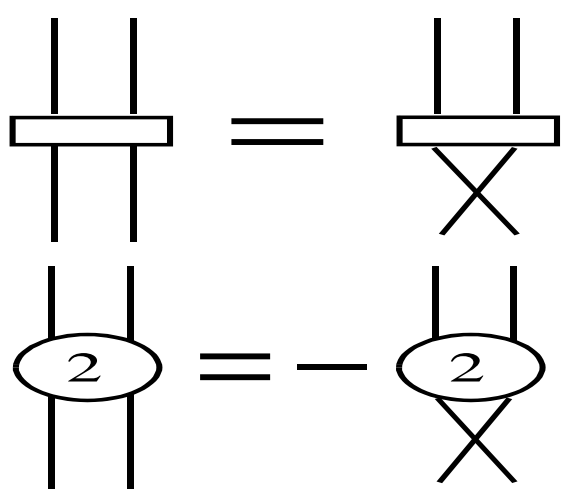

Figure 4: Property I

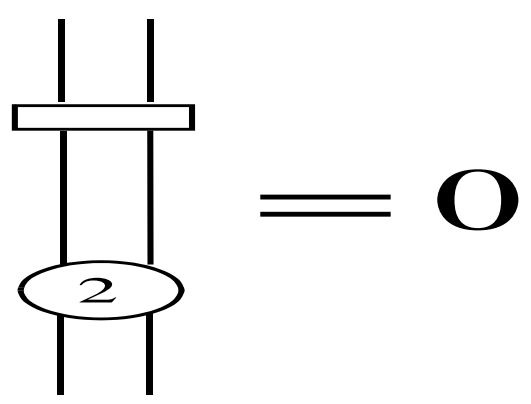

Figure 5: Property II

antisymmetrized at the same time, then obviously they vanishes. Also when one graph involves both boxes and circles, we find the order is also important now. For instance, the graphs shown in fig.(7) are not equivalent.

Continuously taking the antisymmetrization procedures on fundamental representations, we will find it has to be terminated as far as more than $2 n$ fundamental representations involve since antisymmetrizing the same index vanishes. To simplify the notation, we only show one line but label the number of ropes which are anti-symmetrized in the circle. We draw it as fig.(8).

To construct the graphical representation for any highest weight irreducible representation, we need make use of the Young supertableaux. For $O s p(1 \mid 2 n)$, the construction of Young supertableaux is simple since it has the same shape as the usual Young tableaus of $S p(2 n)$ representation $\left(a_{1}, a_{2}, \ldots, a_{n-1}, \frac{1}{2} a_{n}\right)$. The Young tableau associated to $S p(2 n)$ repre- 


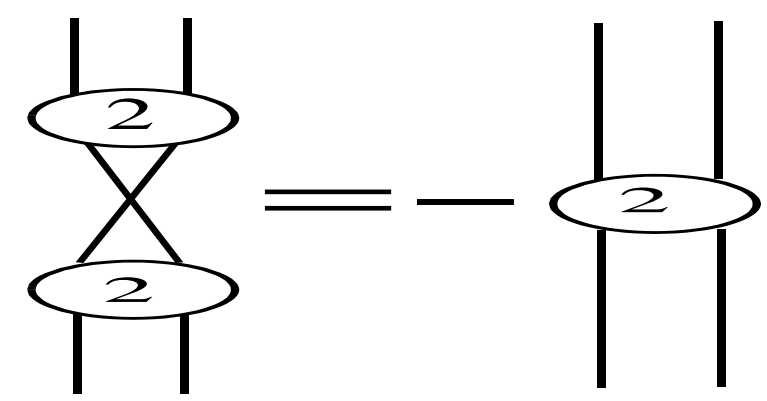

Figure 6: Property III
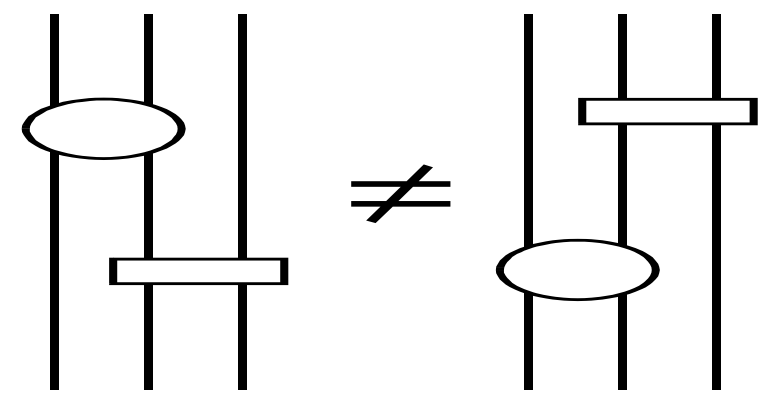

Figure 7: Two distinguished graphs with different orders of box and circle

sentation $\left(a_{1}, a_{2}, \ldots, a_{n-1}, \frac{1}{2} a_{n}\right)$ is defined as a graph with $\lambda_{i}$ boxes in the $i$ th row where $\lambda_{i}$ is related to the Dynkin labels by,

$$
\lambda_{i}=a_{i}+\lambda_{j+1}, \quad \lambda_{n}=\frac{1}{2} a_{n}
$$

which satisfies $\lambda_{1} \geq \lambda_{2} \geq \ldots \geq \lambda_{n} \geq 0$.

Replacing each box in Young supertableaux by a vertical straight rope and putting them in parallel from left to right, then we define a decomposition of the edge by symmetrizing or antisymmetrize them corresponding to their positions in Young supertableaux. As a result, if an irreducible representation is obtained by taking both symmetrization and antisymmetrization procedures, we find there are several inequivalent graphic representations. For example, the representation $(1,1,0,0, \ldots, 0)$ could be realized by graphs illustrated in fig.(9).

In conclusion we find the edge in spin networks can be decomposed into some components which are the combination of some symmetrized or antisymmetrized ropes. An example of 


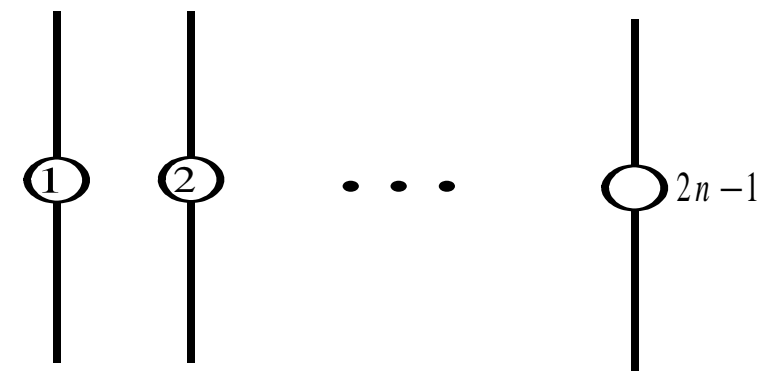

Figure 8: All the non-vanishing antisymmetrizers in $O s p(1 \mid 2 n)$ spin networks

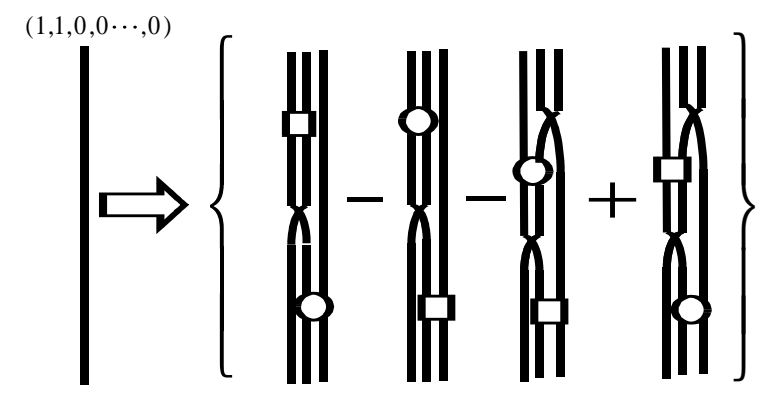

Figure 9: The graphic representation of $(1,1,0, \ldots, 0)$

the decomposition is shown in Fig.(10).

Next we consider the vertex and intertwiners in spin networks. In context of representation theory, it's equivalent to concern the tensor product of irreducible representations. we note that the only Lie superalgebras for which all finite dimensional representations are completely reducible are the direct products of $O s p(1 \mid 2 n)$ superalgebras and semi-simple Lie algebras (Djoković-Hochschild theorem[17]). In context of spin networks, we will have no trouble to connect the edges together at the same vertex. we only need to find out the appropriate admissible conditions and label the vertex by the correct intertwiners, which corresponds to finding the Clebsch-Gordan series for the tensor products of the irreducible representations. To be able to do that, the possible way is to decompose the edge into the direct sum of ones in ordinary $S p(2 n)$ spin networks since the tensor product of two $S p(2 n)$ representations are discussed and the $C G$ coefficients are computable 18. Next let us go back to consider the decomposition of super edges into the ordinary $S p(2 n)$ edges. In the case that only symmetrizations involve, the edge will decomposed into two components, 


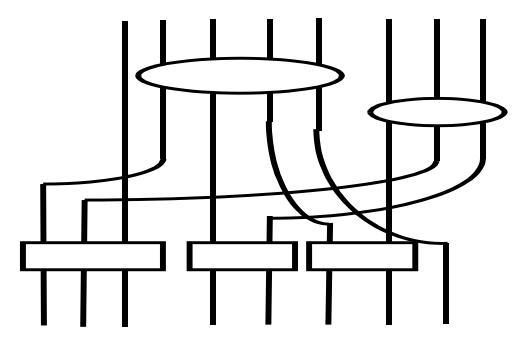

Figure 10: The rope components of general edges

which is illustrated in fig.(11).

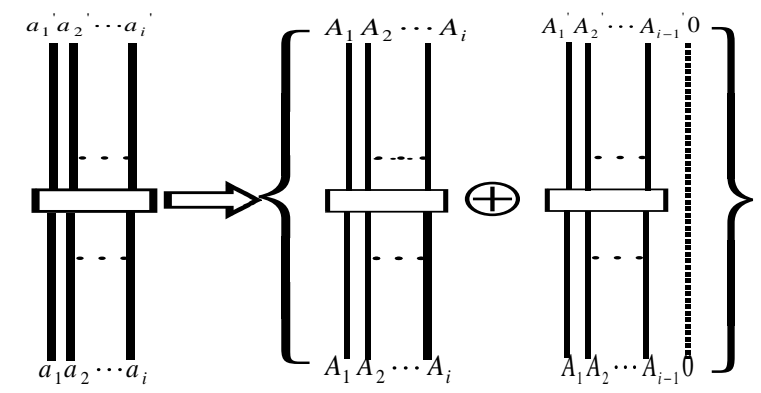

Figure 11: The rope components of general edges

However, if both symmetrization and anti-symmetrizations are involved, then we would find the terms will be more than two, and the specific calculation is possible to carry out. Normally we have the following strategy to obtain all the terms in ordinary $S p(2 n)$ spin networks. The first term in $S p(2 n)$ spin networks has the same shape as the super one, then we divide the ropes into symmetrized groups in which each rope is symmetrized with one another, then pick out at most one rope from each symmetrized group in turn and replace them by the dotted lines such that will get all the graphs in terms of the ordinary $S p(2 n)$ spin networks. Finally we still need kick out any graph which contains at least two dotted but antisymmetrized lines since it vanishes. Among all the graphs remaining, we also have to identify some equivalent graphs and maybe it's a little complicated work. However, in context of Young supertableaux, the procedure is simple. We find the supertableaux of $O s p(1 \mid 2 n)$ can be decompsed into the direct sum of ordinary tableaux by removing at most 
one box from each row. So maybe another practical way is to do the decomposition in context of Young tableaux and then transform the Young diagram into edges of the spin networks. For example, the super one $(1,1,0, \ldots, 0)$ can be decomposed into four $S p(2 n)$ terms as shown in fig.(12).

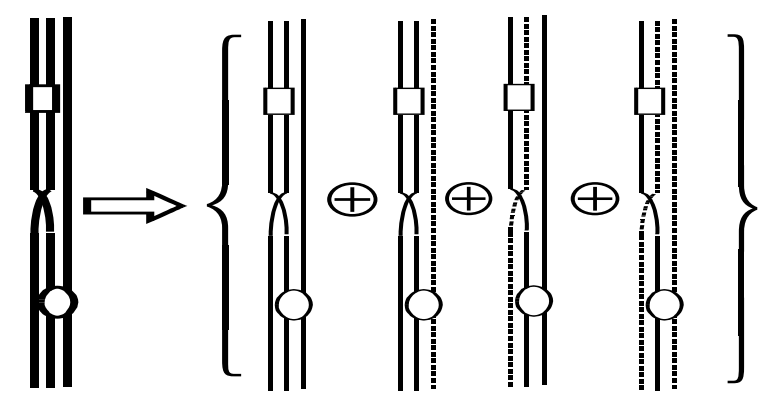

Figure 12: Decomposition of super spin network into ordinary $S p(2 n)$ ones

Then the tensor product of two edges in super spin networks will correspondingly be decomposed to direct sum of the tensor products of edges in $S p(2 n)$ spin networks.

\subsection{Evaluation}

In this paper we haven't carried out a specific calculation for the evaluation of $O s p(1 \mid 2 n)$ spin networks yet. However, based on the following analysis, we would like to claim that it is definitely possible to do so due to the features of $O s p(1 \mid 2 n)$ superalgebra. Basically we have two ways to evaluate such super spin networks. One way is to decompose the super one into the ordinary $S p(2 n)$ spin networks, as we argued above; the other way is given as follows. [15] shows that there is a one-to-one correspondence between the graded representation of $O s p(1 \mid 2 n)$ and the non-spinorial representations of $O(2 n+1)$. First, if a graded irreducible representation of $O \operatorname{sp}(1 \mid 2 n)$ and a non-spinorial irreducible representation of $O(2 n+1)$ have the same highest weight, one has

$$
\operatorname{dim}\left(\rho^{o s p(1 \mid 2 n)}\left(a_{1}, \ldots, a_{n}\right)\right)=\operatorname{dim}\left(\rho^{\prime O(2 n+1)}\left(a_{1}, \ldots, a_{n}\right)\right)
$$

Moreover, the multiplicity of any weight is the same for both representations. As a direct application of the argument, consider the tensor products of both irreducible representations having the same highest weights, then we can see the Clebsch-Gordan $(C G)$ series coincide, 
in particular, since the tensor products are completely reducible, their $C G$ series can be obtained by counting the multiplicities of their weights.

So far, the one-to-one correspondence also give us an alternative practical way to evaluate the $O s p(1 \mid 2 n)$ spin networks by studying the $O(2 n+1)$ spin networks, which should be easy to carry out at first.

In the last part of this section, we conjecture some examples that could be worked out in the future. One is the closure of any edge, namely, the supertrace of the unit element of supergroup $O \operatorname{sp}(1 \mid 2 n)$ in such finite irreducible representation. Note that the supertrace of supergroups is defined as

$$
\operatorname{Str}\left(U_{\beta}^{\alpha}\right):=\operatorname{Tr}(A)-\operatorname{Tr}(D)
$$

where we suppose the matrix representation of the supergroup has a structure,

$$
U=\left(\begin{array}{cc}
A & B \\
C & D
\end{array}\right) .
$$

This can be done by calculating the dimensions of the Lie algebra $O(2 n+1)$ shown in fig.(13).

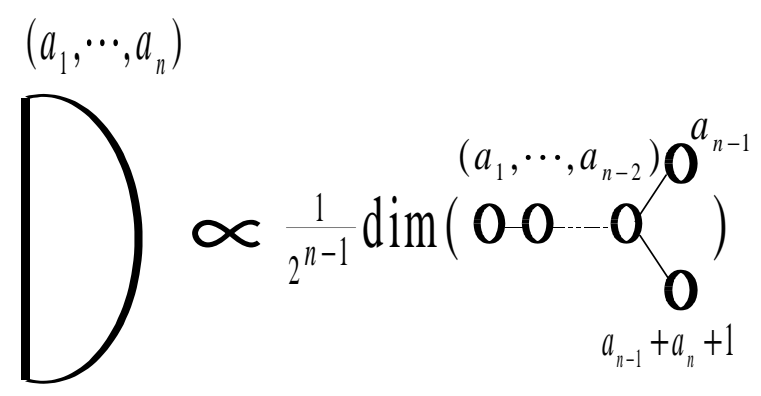

Figure 13: Closure of the edges

We find the closure of the edge is non-zero number. Thus we could expect the identity drawn in fig.(14) is evident. They would also be applied to the non-perturbative quantization of eleven dimensional supergravity when we consider the actions of the operators. For instance, if we consider the quadratic Casimir operators and their eigenvalues?. In loop quantum gravity, this kind of operators is related to the area observable.

$$
C_{k}(\Lambda)=\frac{1}{4 n+2}(\Lambda \mid \Lambda+2 \rho) .
$$

\footnotetext{
${ }^{5}$ There are higher-order Casimir Operators in $O s p(1 \mid 2 n)$.
} 


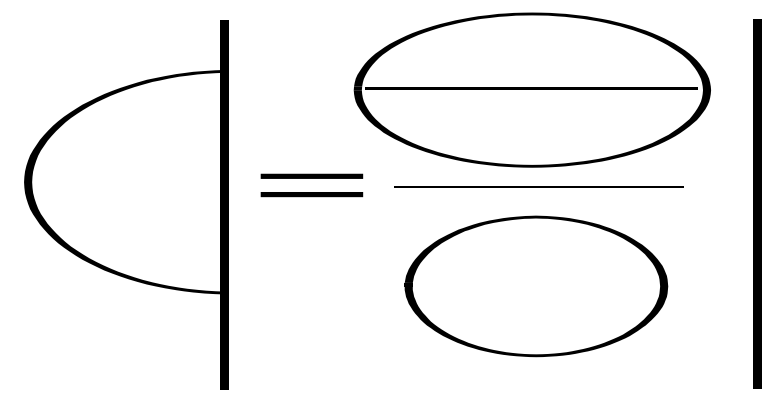

Figure 14: Identity

where $(\mid)$ is defined as the inner product of the weights and $\rho$ is defined as,

$$
\rho=\omega_{1}+\omega_{2} \ldots+\omega_{n} .
$$

The supertrace not being zero has more important meaning when we define super spin network states and form the Hilbert space. This good feature will allow us to define generalized "Ashtekar-Lewandowski" $(A L)$ measure on the space, moreover, to show the spin networks states form a basis of the space.

\subsection{Hilbert Space}

In this part we begin with the integration theory on supergroup manifold, and then apply it to spin network states.

Given a manifold $M$, we define a Lie superalgebra $O s p(1 \mid 2 n)$ valued connection 1-form $A$. For instance, in eleven dimensional space time, we can define the $O s p(1 \mid 32)$ connection as follows,

$$
A=: A_{\mu}(x) d x^{\mu}=A_{\mu}^{a}(x) P_{a} d x^{\mu}+A_{\mu}^{a b}(x) J_{a b} d x^{\mu}+A_{\mu}^{a_{1} \ldots a_{5}}(x) Z_{a_{1} \ldots a_{5}} d x^{\mu}+\Psi_{\mu}^{\alpha}(x) Q_{\alpha} d x^{\mu}
$$

where $x=\left(x_{0}, \ldots, x_{10}\right)$ are local coordinates on $M$, and $\mu$ is space time index and $a, b$

are internal indices. $\alpha$ is spinor index. $P_{a}, J_{a b}, Z_{a_{1} \ldots a_{5}}$, and $Q_{\alpha}$ are $O s p(1 \mid 2 n)$ generators while $J_{a b}$ and $Z_{a_{1} \ldots a_{5}}$ are skew symmetric. In (13) we can think of all the components of connection $A$ as the smooth function on the manifold $M$. Let us denote the space of smooth connections on $M$ as $\mathcal{A}$, then we define the space of continuous functionals on $\mathcal{A}$ as $F u n(\mathcal{A})$. Now based on the linear vector space, we want to define a Hilbert space, $\mathcal{L}^{2}(\mathcal{A})$, namely we 
need introduce some inner product of the quantum states which are the element in $F u n(\mathcal{A})$, and then consider the completion of $F u n(\mathcal{A})$. In loop quantum gravity, we carry out all the procedures by introducing a special kind of functions of the connection, which is called cylindrical functions. They are defined as the functions of holonomies of the connection. Therefore they are functions of group manifold as well. Then the inner products can be defined by means of taking the integration on the group manifold, as we know, a unique, both left- and right-invariant measure can be defined on it.

Now we extend all the construction mentioned above to the supersymmetric case of $O s p(1 \mid 2 n)$. First note the super loop variables can be defined by means of the holonomy of super connections $\mathcal{A}$, for details we refer to 19 .

$$
U[\mathcal{A}, \gamma](s):=\mathcal{P} \exp \int_{\gamma} d s \gamma^{\mu} A_{\mu}(\gamma(s))
$$

Note that $U$ is an element of supergroups $O s p(1 \mid 2 n)$. The loop states can be defined as

$$
\Psi_{\gamma}(A):=\operatorname{Str} U[A, \gamma]
$$

where $\gamma$ is a loop in space time manifold with $\gamma(0)=\gamma(1)$. Though the holonomy is not gauge invariant, but their supertrace is gauge invariant indeed.

It's well known that there is a probability measure, Haar measure, for the compact Lie groups such that we could define a unique normalized both left- and right-invariant integration, namely,

$$
\int_{G} d g=1, \quad \int d g f(g)=\int d g f\left(g_{0} g g_{1}\right)=\int d g f\left(g^{-1}\right),
$$

where $g_{0}, g_{1}$ are any group elements and $f(g)$ is also an arbitrary function of $g$. The generalization of Haar integral for Lie supergroups was discussed in [20]. We refer to that paper for more details on the integration theory on supermanifold. Here we point out that in particular case of $O s p(1 \mid 2 n)$, we can also define a generalized Haar measure on the space of functions on supergroup which are both left- and right-invariant 9 . Therefore it's possible to develop a positive integration theory on the space of connection, $\mathcal{A}$. Here we define the

\footnotetext{
${ }^{6}$ This is also true for all the semisimple Lie supergroups 20, however maybe it does not hold for other supergroups.
} 
generalized Haar measure on supergroup $O s p(1 \mid 2 n)$ as:

$$
\int_{G} d g=1, \quad \int_{G} g_{\alpha \beta}^{(\rho)} d g=0, \rho \neq 0
$$

Next we consider the definition of inner product of spin network states. Associate to every spin network, we can define a corresponding spin network state in the Hilbert space. To show that the set of spin network states does form a basis for the state space in the case of $O \operatorname{sp}(1 \mid 2 n)$, we carry out the following procedures. In spin networks we know a connection is simply to assign a group element, $U_{e}$ to each edge of the graph $e$ by taking the holonomy of the connection $A$ in the irreducible presentation $\rho_{e}$ along the edge. In hence, the space of connections in context of spin networks is

$$
\mathcal{A} \cong \bigotimes_{e_{i}} G^{e_{i}}
$$

which is the finite product of group $G$ and the measure is defined as

$$
\mathcal{D} \mathcal{A}=\bigotimes_{e_{i}} d U_{e_{i}}
$$

We call this measure the generalized Ashtekar-Lewandowski measure. Now we could define the inner product of two spin network states by means of cylindrical functions.

Consider a spin networks $\Gamma\left(e_{i}, v_{j}\right)$, we define the cylindrical functions, $\Phi_{f_{\Gamma}}$, which only depend on the holonomies of the connection $A$. Using the generalized Haar measure on $[G]^{n}$. we then define the scalar product of two cylindrical functions as,

$$
\left\langle\Phi_{f_{\Gamma}} \mid \Phi_{g_{\Gamma}}\right\rangle:=\int_{G^{n}} d U_{1} \ldots d U_{i} \overline{f_{\Gamma}\left(U_{1}, \ldots, U_{i}\right)} g_{\Gamma}\left(U_{1}, \ldots, U_{i}\right) .
$$

The Hilbert space on which $\rho_{l}$ is defined can be denoted as $\mathcal{H}_{\rho_{l}}$. Hence the total Hilbert space associated to the spin networks can be defined as the tensor product of these spaces,

$$
\mathcal{H}=\bigotimes_{v_{j}} \mathcal{H}^{v_{j}}=\bigotimes_{v_{j}}\left(\bigotimes_{e_{i}} \mathcal{H}_{e_{i}}\right)^{v_{j}}
$$

where $e_{i}$ are edges meeting at the same vertex $v_{j}$. In case of $O s p(1 \mid 2 n)$, since any products of finite dimensional irreducible representations are completely reducible, namely,

$$
\rho_{1} \bigotimes \ldots \otimes \rho_{i}=\bigoplus_{j} v^{j} \rho_{j}
$$


correspondingly we find the tensor products of the Hilbert spaces can be decomposed into the direct sum of Hilbert spaces on which the irreducible representation of $O s p(1 \mid 2 n)$ are defined,

$$
\mathcal{H}_{e_{1}} \bigotimes \ldots \bigotimes \mathcal{H}_{e_{i}}=\bigoplus_{\rho_{j}} K^{j} \mathcal{H}_{\rho_{j}}
$$

Furthermore we can decompose the Hilbert space as the direct sum of the functions on all the irreducible representations and its conjugate:

$$
\mathcal{L}^{2}(\mathcal{A} / \mathcal{G})=\bigoplus_{j} V_{j} \bigotimes V_{j}^{*}
$$

Next to show that the spin network states are orthogonal and linear independent, we exploit th generalized Peter-Weyl theorem:

Theorem: let $\rho$ be the irreducible representation of $O s p(1 \mid 2 n)$ with the highest weights $\rho^{i}$, and let $U_{\alpha \beta}^{\rho}, \alpha, \beta=1,2, \ldots d_{i}\left(d_{i}=\operatorname{dim} \rho^{i}\right)$, be the matrix element of $\rho^{i}$, then:

1.

$$
\operatorname{Fun}(A)=\bigoplus_{i} \bigoplus_{\alpha, \beta=1}^{d_{i}} U_{\alpha \beta}^{i}
$$

2.

$$
\int U_{\alpha \beta}^{i} \tilde{U}_{\gamma \sigma}^{j}(-1)^{\beta \gamma+\alpha+\beta}=\delta_{\alpha \gamma} \delta_{i j} \frac{U_{\sigma \beta}^{i}}{\operatorname{Sdim}(i)}
$$

3.

$$
\int U_{\alpha \beta}^{i} \tilde{U}_{\gamma \sigma}^{j}(-1)^{\beta \gamma}=\delta_{\beta \sigma} \delta_{i j} \frac{U_{\alpha \gamma}^{i}}{\operatorname{Sdim}(i)} .
$$

¿From the formula above, we note that the non-zero supertrace plays an important role. However for the supergroups whose supertrace vanishes, then we would maybe have some trouble to find the generalized Peter-Weyl theorem.

Now making use of equations (26) and (27) in the generalized Peter-Weyl theorem, and following the procedures in $\mathbb{4}$, it's straightforward to show the $O \operatorname{sp}(1 \mid 2 n)$ spin states are orthogonal,

$$
\left\langle\Gamma, e^{i}, v_{j} \mid \Gamma^{\prime}, e^{i^{\prime}}, v_{j^{\prime}}\right\rangle=\delta_{\Gamma \Gamma^{\prime}} \delta_{i i^{\prime}} \delta_{j j^{\prime}}
$$

At the same time, using the equation (25) we can show the set of spin network states is completed such that any state in the Hilbert space can be expressed into the sum of the $O \operatorname{sp}(1 \mid 2 n)$ spin network states,

$$
|\Phi\rangle=\sum_{i} C_{i}\left|\Gamma_{i}\right\rangle
$$


Therefore the spin network states do form a basis for the Hilbert space $\mathcal{L}^{2}(\mathcal{A} / \mathcal{G})$.

Finally we point out that in the case of quantum deformed superalgebra, we could also construct the q-deformed spin networks and the corresponding spin network states, which will have important application when we study the casual evolution of the spin networks and supersymmetric spin foams. In the q-deformed case, the ordinary sum of two superspins $J_{1}+J_{2}$ can not give a third superspin any more when q is not equal to one. The notion of co-product has to be introduced. The generalized Peter-Weyl theorem for $U_{q}(\operatorname{ssp}(1 \mid 2 n))$ is given in [21].

In the class of $O s p(1 \mid 2 n)$ spin networks, we are particularly interested in the case of $n=16$, namely $O \operatorname{sp}(1 \mid 32)$, since this superalgebra is related to the eleven dimensional supergravity [22, 23, 24] and $M$ theory [25]. As we know, in eleven dimensional supergravity, the Superpoincare algebra with two and five form central charges are obtained by taking a Inonu-Wigner contraction of $O s p(1 \mid 32)$. This superalgebra has many facets in different dimensions which is studied in [26]. As a result, we expect the $O s p(1 \mid 32)$ spin networks will take its own advantages when we try to carry out a background independent and nonperturbative quantization of the eleven dimensional supergravity, and M theory.

\section{$4 \quad O s p(N \mid 2)$ spin networks}

In previous section we have described $O s p(1 \mid 2 n)$ spin networks following the proposal we give in overview. In this section we will discuss $O s p(N \mid 2)$ spin networks briefly. We are interested in this kind of spin networks because they are related to the chiral supergravities 27. But in this class the case of $N=2$ is different from the others since the even part of this superalgebra is $S O(2) \times S p(2)$ such that the first number $a_{1}$ of Dynkin labels can be any complex number, however for the others it has to be non-negative integer. Its construction and application to supergravity are studies in [11, 28]. Here we consider the general case when $N$ is larger than two.

The basis vectors of the fundamental representation of $O s p(N \mid 2)$ span a $N+2$ dimensional vector space. Similar to the case of $O s p(1 \mid 2 n)$, its higher finite dimensional irreducible representations can be obtained by standard symmetrization and anti-symmetrization procedures

on fundamental representation. Therefore, in context of spin networks, we can decompose 
the edge into ropes.

In fact any representation of basic Lie superalgebra can be decomposed into the direct sum of irreducible representations of the even subalgebra, which means any edge in super spin networks can be decomposed into sum of normal edges which labeled by the irreducible representations of the even subalgebra. In case of $O s p(N \mid 2)$, we can decompose the $O s p(N \mid 2)$ spin networks into the direct sum of the ordinary spin networks with $S O(N) \otimes S p(2)$. In particular, if only the symmetrization procedures are considered, we have simple decompositions. Fig.(15) illustrates the basic one which has the color $(2,0, \ldots, 0)$. Since the $S O(N)$

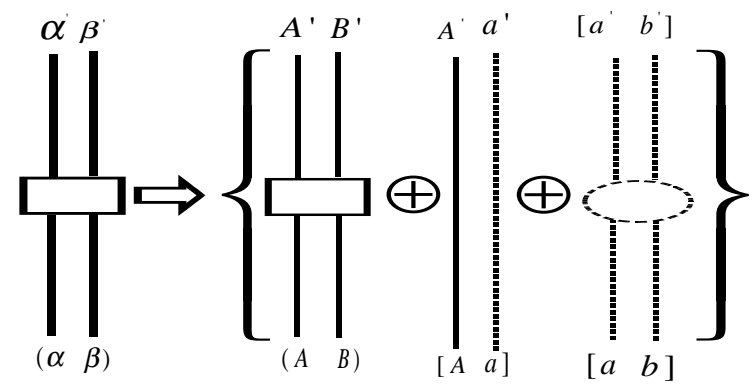

Figure 15: Symmetrizer with color two

indices are antisymmetrized, the decomposition of the graphs terminate at the term which contains $N$ dotted lines, see fig.(16).

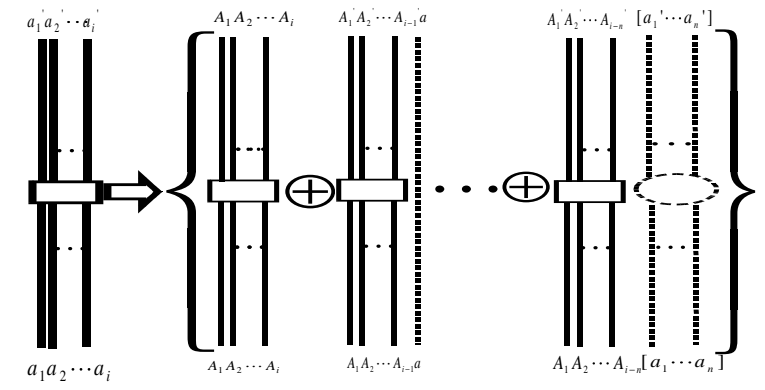

Figure 16: Symmetrizer with color $(i, 0, \ldots, 0)$

However, unlike the case of $O s p(1 \mid 2 n)$, some questions arise in $O s p(N \mid 2)$ case as we try to construct the Hilbert space based on spin network states. As we mentioned before, both typical and atypical representations are present for other superalgebras except $O s p(1 \mid 2 n)$. 
The key difference of these two types of representations is that all the typical representation is either irreducible or completely reducible, however, the atypical one maybe is not completely reducible (i.e., reducible but not decomposable). In group theory, there is a simple meaning of complete reducibility, namely the representation space should be a Hilbert space and the representation should be unitary. Correspondingly in context of spin networks we will meet some serious problems to show the corresponding spin net work states form a basis for the Hilbert space. In this case, we don't know there exists some kinds of generalized Peter-Weyl theorem, though the left-invariant Haar integral for such supergroups are also discussed in [20]. But Let us note there are some classes, which are called star representations and graded star representations in irreducible representations of the superalgebras 14, 29. Inside each class the complete reducibility is reserved indeed and the CG series can be given also. Thus, spanning spin network states in such subspaces, we still expect they could be well defined to form a basis for such Hilbert spaces. Note that in quantum gravity, only the physical Hilbert space is what we want finally. So it's quite interesting to find some self-consistent subset of spin networks which are well defined and investigate the possible applications to quantum theory.

In the end of this section, let us make a summation by listing the correspondence between the construction of spin networks and the representation theory of the superalgebra.

\begin{tabular}{|c|c|}
\hline Spin networks & Representation theory of superalgebra \\
Edges & Irreducible representation \\
Vertex & Intertwiner operator \\
Ropes & Fundamental representations \\
Admissible conditions & Clebsch-Gordan series \\
Closure of edges & Supertrace of the unit element \\
Spin network states & Cylindrical functions \\
Inner product of spin network states & Generalized Haar measure \\
Orthogonality and linear independence of states & Generalized Peter-Weyl theorem \\
\hline
\end{tabular}

\section{Discussions}

In this paper we have presented a general introduction to the construction of supersymmetric spin networks. All the strategy in ordinary $S U(2)$ spin networks can be employed to the case of $O s p(1 \mid 2 n)$ spin networks. In particular the spin network states form a basis for the Hilbert 
space $\mathcal{L}^{2}(\mathcal{A} / \mathcal{G})$. But normally it's becoming more complicated to carry out a practical way to evaluate the spin network graphs, as in general both symmetrization and antisymmetrization procedures are involved in higher finite dimensional irreducible representations. However, it's definitely plausible to give a specific calculation on evaluation of graphs in the future by following strategies given in present paper. On the other hand, due to the properties of the superalgebra, it's unclear if we could construct spin network states as the basis of Hilbert space based on any kind of superalgebra. Here we only discussed the $O s p(N \mid 2)$ spin networks briefly and found this problem should be concerned seriously.

To apply spin-network techniques to quantum supergravities and Yang-Mills theory is very promising and important. At root they are powerful tools to quantize gauge theories along the non-perturbative and background independent approach.

First we expect the corresponding spin network states would be applied to construct the Hilbert space of quantum supergravities. In this framework we could find a practical way to consider the action of the operators, and calculate the spectra of the observables. In [10], we have made a first step to calculate the spectrum of area operator in $N=1$ Chiral supergravity, and the extension to the case of $N=2$ is also done in [11].

Second we propose the holographic hypothesis [30, 31] can be testified in the framework of non-perturbative quantum supergravities. This conjecture has been testifies in $\mathrm{N}=1$ and $\mathrm{N}=2$ supergravity respectively [28, 32]. It is the supersymmetric spin networks that make it possible to count the number of degrees of freedom on the boundary such that we find the relations between the area of the boundary and the number of the states do satisfy Bekenstein's conditions.

Until now we only take into account for the finite dimensional representation of the superalgebra. It is worth studying what role the spin networks will play if we consider the infinite dimensional representation of the superalgebra.

\section{Acknowledgement}

I am very grateful to my advisor, Prof. Lee Smolin, for his contribution and encouragement during the course of this work. Also I'd like to thank Laurent Freidel, Marcus Gaul, Yongge Ma and Daniele Oriti for their continuous interests in this subject and communication. I am 
also grateful to the theoretical physics group at Imperial College for hospitality when part of work was done there. This work was supported by the NSF through grant PHY95-14240 and a gift from the Jesse Phillips Foundation.

\section{References}

[1] R. Penrose, in Quantum theory and beyond, ed T Bastin, Cambridge U Press, 1971.

[2] J. Kogut and L. Susskind, Phys. Rev. D11 (1975)395; W. Furmanski and A. Kowala, Nucl. Phys. B291(1987)594. J. Baez, Adv.Math. 117 (1996) 253.

[3] E. Witten, Commum. Math. Phys. 121(1989)351; V. Turaev and O. Viro, Topology 31(1992) 865; H. Ooguri, Mod. Phys. Lett A7(1992)2799; L. Crane and D. Yetter, in Quantum Topology, eds L. H. Kauffman and R. A. Baadhio, World Scientific Press, (1993) 120; L. Crane and I. B. Frenkel, J. Math. Phys. 35(1994)5136; L. Crane and D. Yetter, On algebra structure implicit in topological quantum field theories, Kansas preprint 1994; T. J. Foxon, Class.Quant.Grav. 12 (1995) 951; C. Rovelli, Phys. Rev. D48 (1993) 2702; S. Mizoguchi and T. Tada, Phys. Rev. Lett. 68 (1992) 1795.

[4] C. Rovelli and L. Smolin, Phys. Rev. D52 (1995) 5743; J. Baez, Spin networks in nonperturbative quantum gravity, in The Interface of Knots and Physics, ed. Louis Kauffman, A.M.S., Providence, 1996, 167; A. Ashtekar, J. Lewandowski, D. Marolf, J. Mourao, T. Thiemann, J. Math. Phys. 36 (1995) 6456.

[5] A Ashtekar, J Lewandowski, D Marlof, J Mourãu and T Thiemann, Quantization of diffeomorphism invariant theories of connections with local degrees of freedom, gr-qc/9504018, JMP 36 (1995) 519; A. Ashtekar and J. Lewandowski, Quantum Geometry I: area operator, gr-qc/9602046; J. Lewandowski, Volume and quantization, gr-qc/9602035; T. Thiemann, Quantum Spin Dynamics I-VI, Class. Quant. Grav. 15 (1998) 839-873, 875-905, 1207-1247, 1249-1280, 1281-1314, 1463-1485, 14871512. gr-qc/9606092, gr-qc/9606089, gr-qc/9606090, gr-qc/9705020, gr-qc/9705021, grqc/9705019, gr-qc/9705018, gr-qc/9705017. 
[6] C. Rovelli and L. Smolin Discreteness of area and volume in quantum gravity Nuclear Physics B 442 (1995) 593. Erratum: Nucl. Phys. B 456 (1995) 734.

[7] F. Markopoulou, Dual formulation of spin network evolution preprint, March 1997, gr-qc/9704013; F. Markopoulou and L. Smolin Causal evolution of spin networks grqc/9702025. CGPG preprint (1997), Nuclear Physics B, Nucl.Phys. B508 (1997) 409430; F. Markopoulou and L. Smolin Quantum geometry with intrinsic local causality, Phys. Rev. D58 (1998) 084032, gr-qc/9712067.

[8] M. Reisenberger, A lattice worldsheet sum for 4-d Euclidean general relativity, grqc/9711052; M. Reisenberger and C. Rovelli, "Sum over Surfaces" form of Loop Quantum Gravity, Phys.Rev. D56 (1997) 3490, gr-qc/9612035; J. Baez,Spin foam models, Class.Quant.Grav. 15 (1998) 1827, gr-qc/9709052; J. Baez, An Introduction to Spin Foam Models of Quantum Gravity and BF Theory, gr-qc/9905087.

[9] J. Barrett and L. Crane, Relativistic spin networks and quantum gravity, J. Math. Phys. 39 (1998) 3296, gr-qc/9709028; A Lorentzian Signature Model for Quantum General Relativity Class. Quant. Grav. 17 (2000) 3101, gr-qc/9904025; S. Davids, Semiclassical Limits of Extended Racah Coefficients, J. Math. Phys. 41 (2000) 924, gr-qc/9807061; M. Bojowald, Abelian BF-Theory and Spherically Symmetric Electromagnetism, J. Math. Phys. 41 (2000) 4313, hep-th/9908170.

[10] Y. Ling and L. Smolin, The supersymmetric spin networks and quantum supergravity, Phys. Rev. D61, 044008(2000), hep-th/9904016.

[11] Y. Ling and L. Smolin, $N=2$ quantum supergravity, preprint in preparation.

[12] L. Kauffman, S. Lins, Temperley-Lieb Recoupling Theory and Invariants of 3-Manifolds, Princeton U Press, 1994.

[13] V. Kac, Adv. Math.26, (1977)8; Commun. Math. Phys. 53(1977)33; Lecture Notes in Mathematics vol.676( New York, Springer).

[14] L. Frappat, A. Sciarrino and P. Sorba, Dictionary on Lie Superalgebras, hep-th/9607161. 
[15] V. Rittenberg and M. Scheunert, A Remarkable Connection Between the Representations of the Lie Superalgebras osp(1, 2n) and the Lie Algebra O(2n+1), Commun. Math. Phys. 83, 1 9(1982).

[16] A. Balantekin and I. Bars, Dimension and character formulas for Lie supergroups, J. Math. Phys.22,(1981)1149; Representation of supergroups, J. Math. Phys.22,(1981)1810.

[17] G. Hochschild, III, J. Math. 20, 107(1976); D.Djoković and G. Hochschild, III, J. Math.20, 134(1976); D. Djoković, J. Pure Appl. Alg. 7, 217(1976).

[18] B. More, A. Sciarrino and P. Sorba, Representation of the $O \operatorname{sp}(M \mid 2 n)$ and Young supertableaux, J. Phys. A: Math. Gen. 18(1985)1597. G. Girardi, A. Sciarrino and P. Sorba, Kronecker product of Sp(2n) representations using generalised Young tableaux, J. Phys. A: Math. Gen. 16(1983)2609.

[19] L. Urrutia, Towards a loop representation of connection theories defined over a super Lie algebra, aipproc style Based on the lectures given at the Fifth Workshop on Particles and Fields, Puebla, Nov. 1995, hep-th/9609001.

[20] D. Williams and J. F. Cornwell, Haar Integral for Lie supergroups, J. Math. Phys. $25(1984) 2922$.

[21] H. C. Lee and R. B. Zhang, Geometry and representations of the quantum supergroup $O \operatorname{sp}_{q}(1 \mid 2 n)$, math.QA/9804111; M. Scheunert, R. B. Zhang Invariant integration on classical and quantum Lie supergroups, math.RA/9911200.

[22] L. Castellani, P. Fre and P. van Nieuwenhuizen, "A Review Of The Group Manifold Approach And Its Application To Conformal Supergravity," Annals Phys. 136, 398 (1981).

[23] R. D'Auria and P. Fre, "Geometric Supergravity In D = 11 And Its Hidden Supergroup," Nucl. Phys. B201, 101 (1982). Erratum-ibid.B206:496,1982.

[24] Y. Ling and L. Smolin, Eleven dimensional supergravity as a constrained topological field theory, hep-th/0003285. 
[25] P. K. Townsend, M theory from its superalgebras, Cargese lectures 1997, hep-th/9712004; M. Gunaydin, Unitary supermultiplets of $O \operatorname{sp}(1 / 32, R)$ and $M$ theory, Nucl.Phys. B528 (1998) 432-450, hep-th/9803138.

[26] E. Bergshoeff, A. Proeyen, The many faces of $O S p(1 \mid 32)$, Class.Quant.Grav. 17 (2000)3277, hep-th/0003261.

[27] T. Jacobson, New variables for canonical supergravity Class. Quant. Grav. 5 (1988) 923; D. Armand-Ugon, R. Gambini, O. Obregon, J. Pullin, Towards a loop representation for quantum canonical supergravity, hep-th/9508036, Nucl. Phys. B460 (1996) 615; H. Kunitomo and T. Sano The Ashtekar formulation for canonical N=2 supergravity, Prog. Theor. Phys. suppl. (1993) 31; Takashi Sano and J. Shiraishi, The Non-perturbative Canonical Quantization of the N=1 Supergravity, Nucl. Phys. B410 (1993) 423, hepth/9211104; The Ashtekar Formalism and WKB Wave Functions of N=1,2 Supergravities, hep-th/9211103; K. Ezawa, Ashtekar's formulation for $N=1, N=2$ supergravities as constrained BF theories, Prog. Theor. Phys.95:863-882, 1996, hep-th/9511047.T. Kadoyoshi and S. Nojiri, $N=3$ and $N=4$ two form supergravities, Mod. Phys. Lett. A12:1165-1174,1997, hep-th/9703149; L. F. Urrutia Towards a loop representation of connection theories defined over a super-lie algebra hep-th/9609010.

[28] Y. Ling and L. Smolin, Holography, BPS states and N=2 quantum supergravity, preprint in preparation.

[29] M.Scheunert, W.Nahm, and V.Rittenberg, Graded Lie Algebras: Generalization of Hermitian representations, J. Math. Phys.18(1977)146; Irreducible representations of the osp (2,1) and $\operatorname{spl}(2,1)$ graded Lie algebras, J.Math.Phys.18,(1977)155.

[30] L. Smolin, Linking topological quantum field theory and nonperturbative quantum gravity,gr-qc/9505028, J. Math. Phys. 36(1995)6417;

[31] L. Smolin, A holograpic formulation of quantum general relativity, hep-th/9808191.

[32] Y. Ling and L. Smolin, Holographic formulation of $N=1$ supergravity, preprint in preparation. 